\title{
The Implications of Alternative Allocation Criteria in Adaptive Design for Panel Surveys
}

\author{
Olena Kaminska ${ }^{1}$ and Peter Lynn ${ }^{1}$
}

\begin{abstract}
Adaptive survey designs can be used to allocate sample elements to alternative data collection protocols in order to achieve a desired balance between some quality measure and survey costs. We compare four alternative methods for allocating sample elements to one of two data collection protocols. The methods differ in terms of the quality measure that they aim to optimize: response rate, R-indicator, coefficient of variation of the participation propensities, or effective sample size. Costs are also compared for a range of sample sizes. The data collection protocols considered are CAPI single-mode and web-CAPI sequential mixedmode. We use data from a large experiment with random allocation to one of these two protocols. For each allocation method we predict outcomes in terms of several quality measures and costs. Although allocating the whole sample to single-mode CAPI produces a higher response rate than allocating the whole sample to the mixed-mode protocol, we find that two of the targeted allocations achieve a better response rate than single-mode CAPI at a lower cost. We also find that all four of the targeted designs out-perform both single-protocol designs in terms of representativity and effective sample size. For all but the smallest sample sizes, the adaptive designs bring cost savings relative to CAPI-only, though these are fairly modest in magnitude.
\end{abstract}

Key words: Coefficient of variation; effective sample size; mixed mode; optimal allocation; R-indicator; survey costs.

\section{Introduction}

At the heart of adaptive design lies the idea that survey method can be adapted better to suit different subgroups of sampled persons (elements). This perspective brings new opportunities for exploring methods for improving survey quality. A large body of previous methodological research has addressed questions of whether one survey strategy (or protocol) is better than another in terms of some desired outcome(s) such as response rate, sample composition, survey costs, measurement validity, or measurement precision. Such studies focus on the average effect, across the sample, of the protocol on the desired outcome. These studies are therefore informative regarding which protocol is likely to perform better if it is applied to everyone in a sample. However, it can be the case that even

\footnotetext{
${ }^{1}$ ISER, University of Essex, Wivenhoe Park, Colchester, CO4 3SQ, UK. Emails: olena@essex.ac.uk and plynn@essex.ac.uk.

Acknowledgments: The authors are funded by a research award from the UK Economic and Social Research Council (award no. ES/K005146/1) for "Understanding Society: the UK Household Longitudinal Study, phase 3." The successful design and implementation of Wave 5 of the Understanding Society Innovation Panel, our source of data, was due to the diligence of many colleagues at ISER, University of Essex, and at NatCen Social Research, who carried out the data collection.
} 
better outcomes could be achieved if some subgroups of the sample were instead assigned to a protocol with a 'worse' average effect. That would be the case if effects were heterogeneous across sample subgroups in such a way that a protocol with a worse average effect was nevertheless the optimum strategy for one or more subgroups. A close look at the survey methodology literature suggests that this may often be the case. For example, studies have found effects on response rates to be heterogeneous across sample subgroups for the following design features: respondent incentives (Singer and Ye 2013), design of survey invitation mailings (Kaplowitz et al. 2012), mode of approach (Kaplowitz et al. 2004), survey topic (Sheehan 2001), and interviewer characteristics (Durrant et al. 2010). In other words, in the same way that advertisers benefit from targeting online adverts based on personal web browsing information, survey researchers may be able to benefit from targeting survey protocols based on some relevant information about survey sample elements.

Improving desirable survey outcomes through adaptive designs depends on the ability to effectively allocate sample elements to alternative protocols. Important questions therefore concern the criteria that should be used to allocate sample elements to different available protocols and the methods that should be used to implement these criteria. The choice of criterion will depend on which survey outcome(s) the researcher wants to optimize. If suitable information is available about sample elements before data collection starts, static adaptive designs (Bethlehem et al. 2011) or targeted designs (Lynn 2014) can be employed. If such information is not available it may be possible to learn about sample elements during data collection (Durrant et al. 2015) and adapt the design using the initial information during the fieldwork in a dynamic adaptive design (Bethlehem et al. 2011) or responsive design (Groves and Heeringa 2006). This article considers static adaptive designs in which sample allocation is based on information available before fieldwork, in the manner of Lynn (2017).

Most of the literature on adaptive design considers nonresponse error as the outcome of interest, with one notable exception which looks at measurement error (Calinescu and Schouten 2015). Yet there is no one agreed measure of quality with respect to nonresponse error, and currently research explores different quality indicators that can be optimized through adaptive design. A broad review of such indicators is presented by Groves et al. (2008), and a typology is suggested by Wagner (2012). Many quality indicators are itemdependent, that is, their value differs across survey items and one or a few important items need to be selected for optimization. These include estimated nonresponse bias (Groves and Heeringa 2006) and the fraction of missing information (Wagner 2008). Furthermore, balance indicators which measure the balance of the response set with regard to means of some important items (Särndal 2011; Lundquist and Särndal 2013) also depend on the selection of survey items.

This article considers quality indicators which have one value for the whole survey and measure "the degree to which the respondents to a survey resemble the complete sample" (Schouten et al. 2011, 2). As alternatives to the response rate different quality indicators have been developed to measure the general representativity of survey participants. These include the R-indicator (Schouten et al. 2009; Schouten et al. 2012; Shlomo et al. 2013); variance functions of nonresponse weights (Groves et al. 2008; Bethlehem et al. 2011); maximal bias and maximal mean square error (Bethlehem et al. 2011), among others. 
More complex optimization exercises include constraining minimum sample size and/or cost while simultaneously optimizing one of the above quality indicators (Schouten et al. 2013; Schouten and Shlomo 2015; Calinescu and Schouten 2015).

While the debate on different quality indicators continues, this article explores related questions: 1) how can survey samples be allocated to data collection protocols according to different allocation criteria? 2) how different will the allocation be depending on which criterion is applied? 3) how will outcomes vary between the allocation scenarios? 4) can adaptive designs out-perform one-protocol-for-all designs in terms of these outcomes? Specifically, we look at an allocation to one of two protocols (CAPI single-mode and webCAPI sequential mixed-mode) in the context of a panel survey. This context is useful as there is rich information on panel participants at any point after Wave 1. This information can be used to inform the allocation procedures, as we show below, and can also serve as auxiliary data to evaluate the outcomes of an adaptive design. However, our approach should be more broadly applicable to any situation where suitable information is available before the fieldwork and two or more protocols are available to choose from.

We structure our article in the following way. First we describe our data and the context in which allocation is required. We follow with a description of the allocation criteria based on alternative quality indicators and of the allocation methods and cost estimate method. The following section presents the resultant allocation to protocols for each scenario and evaluates the scenarios using different outcome indicators, including both quality indicators and cost estimates. We conclude by discussing the potential for adaptive design in practice, the role of different quality indicators in this context, and possible future advances in allocation criteria.

\section{Data: UK Household Longitudinal Study Innovation Panel}

Our data for this study comes from Innovation Panel (IP) which is a standalone mediumscale probability-based panel designed specifically to inform a large panel, the UK Household Longitudinal Study (UKHLS), through experimentation (Burton 2013). This setup is especially advantageous for adaptive design as it allows measurement of participants' response to different strategies before they are implemented on the mainstage UKHLS. IP started in 2008, one year before UKHLS, and has included many experiments that have already informed the mainstage procedures (Burton 2013; Al Baghal 2014; Al Baghal 2015; Lynn and Jäckle, in press).

In this article we explore data from a mixed mode experiment implemented in Wave 5 of IP, with field work carried out from 11 May to 5 September 2012. While IP is primarily CAPI in previous waves, at Wave 5 one random third of the sample was allocated to a CAPI single-mode protocol and the remaining two random thirds were allocated to a mixed-mode protocol (web, followed by CAPI for nonrespondents). The mixed-mode (MM) protocol included a postal letter with an invitation to complete a survey via web; for those with email addresses the same letter was also sent by email with two subsequent reminders (or one postal reminder if no email address was known). After two weeks all adults who had not yet filled the questionnaire online were issued into CAPI mode (Burton 2013), meaning that interviewers began visiting the sample addresses to seek face-to-face interviews. The CAPI phase of the field work lasted 14 weeks. The total response rate 
conditional on participation in Wave 4 was $81.2 \%$, with the mixed-mode protocol obtaining $79.4 \%$ (of which $39.9 \%$ responded via web mode) and CAPI protocol obtaining $84.6 \%$ (Jäckle et al. 2015). Further details of the survey design and implementation can be found in White et al. (2011).

The main aim of the experiment was to inform the mainstage UKHLS on how to best allocate participants to these protocols, as part of the study is switching from CAPI singlemode to a mixed-mode protocol. While the study interviews everyone aged over ten in each household and the allocation is at the household level (and thus more complex) and has budget restrictions, for the purpose of this article we treat individual sample persons as the elements to be allocated to protocols and do not explicitly take into account cost in the allocation criteria. Our study is restricted to sample elements who participated in the survey at Wave 4 and the objective is to shed light on some of the issues involved in implementing allocation procedures to optimize different quality criteria for nonresponse, including the evaluation of cost estimates.

\section{Allocation to Optimize Quality Criteria}

In this section we describe the four different quality criteria that we use to inform sample allocation and the method that we use to implement the allocation in each case. In all cases the allocation method draws on predicted probabilities of participation under each data collection protocol. The first step is therefore to estimate probabilities of participating in the CAPI single-mode protocol and separately in the mixed-mode protocol for each sample element conditional on participation in the previous wave. To do this we use general demographic and substantive variables from IP Wave 4 as predictors, selected as being either theoretically related to nonresponse (Groves and Couper 1998), empirically associated with panel attrition (Uhrig 2008; Watson and Wooden 2009), or observed to distinguish between web and CAPI response in a mixed-mode context (Jäckle et al. 2015). These include age, gender, area type, ethnicity, whether born in the UK or outside, marital status, religion, employment status, personal income, savings, self-rated health, BMI, household size, government region, presence of children under ten in the household, ownership of the house, number of rooms per person, amenities, whether lone parent, presence of parents in the household, whether mobile phone owner, whether a carer, whether respondent has a driving licence, whether likely to move in the upcoming year, whether others were present at last interview, influence of others on previous interview, interviewer assessment of whether respondent was cooperative at the last interview, whether there is a computer in the house, number of cars owned, whether up to date with all bills, whether can afford holiday, and internet usage. The Wave 4 data collection protocol was CAPI-only for all sample elements, so the variables regarding the interview context have a consistent interpretation across both Wave 5 experimental groups, and there is no mode-induced differential measurement. We use the same set of covariates for all three models in order to avoid differences between protocols in predicted probabilities being due to omitted variables. We note, however, that some variables may play an important role in one model while having little influence in another. The advantage for adaptive design is that not only do we have very rich information about our panel members 
but also it is highly related to the variables of interest as mostly the predictors are lagged versions of the variables measured in the following wave.

With the above predictors, using logistic regression we predict three propensities: the probability of participating, (a) in the CAPI single-mode protocol (based on the CAPI experimental sample), (b) in the mixed-mode protocol (based on the mixed-mode experimental sample), and (c) on the web (based on the mixed mode experimental sample). All three models are conditional on participation in the previous wave. Although web participation is part of the overall mixed-mode participation, it is of particular interest as high web participation is desired for cutting costs of data collection (see Section 4 below). Model-predicted values are then calculated for all three models for both experimental groups. Thus, for all sample elements, regardless of their experimental assignment, we obtain three predicted propensities, denoted as follows:

$p_{i j}$ is the probability of element $i$ participating if allocated to protocol $j$;

$j=1$ indicates CAPI single-mode protocol;

$j=2$ indicates mixed-mode protocol;

$q_{i 2}$ is the probability of element $i$ participating in web mode if allocated to protocol 2; $i=1 \ldots n$, where $n$ is the total number of Wave 4 respondents who were issued to the field for Wave 5 .

Note that $q_{i 2} \leq p_{i 2} \forall i$.

The probabilities $\left\{p_{i j}\right\}$ will be used at the next step to determine the allocation of each sample element to a data collection protocol under each allocation scenario, and all three probabilities will be used at the subsequent step (Section 5) to predict the outcomes of each allocation scenario. Details of the three fitted models are summarized in Table 1. It can be observed that some variables appear to perform a similar role in each model, suggesting that they are broadly predictive of survey participation regardless of the data collection mode (for example age, wealth and body mass index), while others have different coefficients in each model, suggesting a mode-specific association with participation (for example, rurality, marital status and the presence of children in the household). Figure 1 shows the association between $p_{i 1}$ and $p_{i 2}$ (the probability of element $i$ responding in CAPI single-mode and mixed-mode protocol respectively) and demonstrates that the two models provide considerable discrimination: for any value of $p_{i 2}$ there is considerable variation in the values of $p_{i 1}$.

We consider seven different allocation scenarios. Three of these can be considered as benchmark designs and are described in the next paragraph. The other four are static adaptive designs, each involving allocation to data collection protocols based on a different criterion. The allocation criteria for the adaptive designs and the method used to implement them are described in Subsections 3.1 to 3.4 respectively. For each of the allocation scenarios the allocated protocol can be indicated as follows:

$$
I_{i j k}=1 \text { if element } i \text { is allocated to protocol } j \text { under scenario } k \text {, for } k=1 \text { to } 7 \text {. }
$$

Note that: $\sum_{j=1}^{2} I_{i j k}=1 \forall(i, k)$, that is, under each scenario, each element is allocated uniquely to one protocol.

Consequently, the response propensity of element $i$ in scenario $k$ will be $\rho_{i k}=\sum_{j=1}^{2} I_{i j k} p_{i j}$. 
Table 1. Predictive models of participation under CAPI protocol, mixed mode protocol and web-only protocol; Standardised coefficients and standard errors.

\begin{tabular}{|c|c|c|c|}
\hline & $\begin{array}{l}\text { CAPI prob } \\
\quad\left(p_{i 1}\right)\end{array}$ & $\begin{array}{l}\text { MM prob } \\
\quad\left(p_{i 2}\right)\end{array}$ & $\begin{array}{l}\text { Web prob } \\
\left(q_{i 2}\right)\end{array}$ \\
\hline Age & $0.09(0.05)$ & $0.04(0.03)$ & $0.04(0.03)$ \\
\hline Age squared & $-0.00(0.00)$ & $-0.00(0.00)$ & $-0.00(0.00)$ \\
\hline Rural area & $0.60(0.35)$ & $-0.52(0.18)$ & $-0.45(0.16)$ \\
\hline Carer & $1.06(0.54)$ & $0.10(0.27)$ & $-0.03(0.25)$ \\
\hline Has driving licence & $-0.14(0.30)$ & $-0.46(0.18)$ & $-0.34(0.18)$ \\
\hline \multicolumn{4}{|l|}{ Government Region $(\operatorname{Ref}=$ North $)$ : } \\
\hline Midlands & $0.49(0.37)$ & $-0.09(0.23)$ & $-0.16(0.20)$ \\
\hline East Anglia & $0.30(0.45)$ & $-0.01(0.29)$ & $0.30(0.24)$ \\
\hline London & $0.07(0.45)$ & $-0.81(0.28)$ & $-0.14(0.27)$ \\
\hline South & $0.38(0.34)$ & $-0.23(0.20)$ & $0.01(0.18)$ \\
\hline Wales & $0.13(0.52)$ & $0.61(0.40)$ & $-0.05(0.34)$ \\
\hline Scotland & $-0.45(0.45)$ & $-0.39(0.27)$ & $0.11(0.26)$ \\
\hline \multicolumn{4}{|c|}{ Marital status $($ Ref $=$ never married $)$ : } \\
\hline Married/civil partnership & $-0.57(0.41)$ & $0.13(0.25)$ & $0.64(0.23)$ \\
\hline Widowed/divorced/separated & $0.26(0.49)$ & $-0.35(0.27)$ & $-0.17(0.26)$ \\
\hline Belongs to a religion & $0.06(0.26)$ & $0.27(0.16)$ & $0.31(0.14)$ \\
\hline \multicolumn{4}{|l|}{ Interest from savings $(\operatorname{Ref}=$ none $)$ : } \\
\hline Up to $£ 500$ & $0.23(0.34)$ & $0.25(0.21)$ & $0.39(0.17)$ \\
\hline Over $£ 500$ & $0.18(0.61)$ & $0.74(0.34)$ & $0.73(0.28)$ \\
\hline Others influenced interview & $-0.47(0.42)$ & $-0.53(0.27)$ & $-0.09(0.26)$ \\
\hline \multicolumn{4}{|l|}{ Co-operative $(\operatorname{Ref}=\mathrm{v}$ good $):$} \\
\hline Good & $0.24(0.33)$ & $-0.24(0.18)$ & $-0.16(0.17)$ \\
\hline Fair/poor/very poor & $0.20(0.63)$ & $-0.72(0.36)$ & $-0.88(0.49)$ \\
\hline Body mass index $\geq 28$ & $-0.30(0.26)$ & $-0.35(0.16)$ & $-0.25(0.15)$ \\
\hline Number of children aged under 10 & $0.38(0.35)$ & $-0.27(0.23)$ & $-0.48(0.21)$ \\
\hline Internet access at home & $0.42(0.46)$ & $0.45(0.29)$ & $1.65(0.35)$ \\
\hline \multicolumn{4}{|c|}{ Number of cars in household $(\operatorname{Ref}=0)$ : } \\
\hline One & $0.86(0.41)$ & $-0.30(0.25)$ & $-0.19(0.27)$ \\
\hline Two or more & $0.58(0.50)$ & $0.09(0.30)$ & $0.26(0.30)$ \\
\hline Behind with paying bills & $-0.79(0.43)$ & $0.08(0.31)$ & $-0.35(0.34)$ \\
\hline \multicolumn{4}{|l|}{ Material deprivation indicator: } \\
\hline Cannot afford holiday & $0.32(0.33)$ & $0.05(0.20)$ & $-0.35(0.19)$ \\
\hline Does not want holiday & $-0.38(0.38)$ & $-0.02(0.24)$ & $-0.45(0.22)$ \\
\hline Regularly uses internet & $-0.45(0.40)$ & $0.24(0.25)$ & $0.89(0.24)$ \\
\hline$R^{2}$ & 0.13 & 0.09 & 0.20 \\
\hline$n$ & 735 & 1,374 & 1,374 \\
\hline Mean predicted probability & 0.85 & 0.80 & 0.41 \\
\hline Std. Dev. & 0.13 & 0.13 & 0.24 \\
\hline Skewness & -1.73 & -1.06 & -0.11 \\
\hline Kurtosis & 6.56 & 4.00 & 2.00 \\
\hline
\end{tabular}

Note: Each model included same set of 32 variables, as explained in the text; table shows only variables with $\mathrm{P}<0.05$ for at least one category in at least one model. 


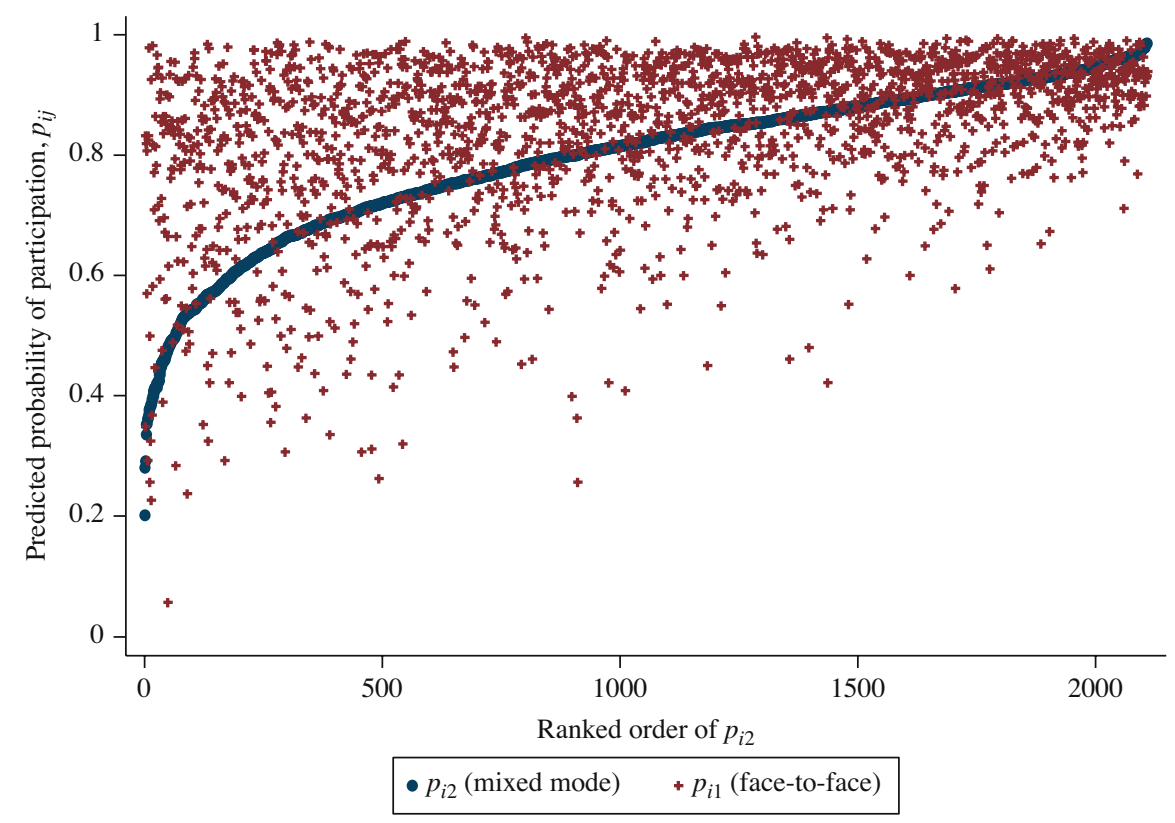

Fig. 1. Association of predicted probabilities of participation under two data collection protocols.

For comparative purposes we consider three benchmark allocation scenarios. Two of these assume that all participants are assigned either to the CAPI single-mode protocol ('CAPI-for-all') or to the mixed-mode protocol ('MM-for-all'). Thus, under CAPI-for-all $(k=1), \quad I_{i 11}=1$ and $I_{i 21}=0 \forall i$, while under MM-for-all $(k=2), I_{i 12}=0$ and $I_{i 22}=1 \forall i$. The third scenario $(k=3)$ reflects the random allocation from the actual experiment which assigned two-thirds of the sample to the mixed-mode protocol and onethird to the CAPI single-mode protocol ('experimental allocation'). Unlike for other allocations the composition of the responding sample is observed (rather than being simulated using model-predicted probabilities).

\subsection{Response-Rate (RR) Optimization}

Survey researchers have for decades striven to achieve the highest possible response rates (Kanuk and Berenson 1975; Sheehan 2001; Yu and Cooper 1983). Many of the early static adaptive designs allocated sample elements to treatments with the objective of maximizing response rate (Lynn 2017). We therefore include amongst the set of adaptive designs that we study, one $(k=4)$ which aims to maximize the response rate. To do this, we allocate each element according to their highest predicted response propensity. Thus for each element we compare the response propensities for the CAPI single-mode and mixed-mode protocols and base the allocation on the higher of the two. Hence:

$$
\begin{array}{lll}
I_{i 14}=1 \quad \text { and } & I_{i 24}=0 & \text { if } \quad p_{i 1}>p_{i 2} ; \\
I_{i 14}=0 & \text { and } \quad I_{i 24}=1 & \text { otherwise. }
\end{array}
$$




\subsection{R-Indicator Optimization}

Increasing the response rate does not necessarily decrease nonresponse error (Groves and Peytcheva 2008). Thus we explore an allocation $(k=5)$ that aims to improve representativity. In other words we aim to allocate sample elements to protocols such that the expected distribution of selected variables among respondents is as close to their distributions for the gross (issued) sample as possible. We optimize to the R-indicator in its simplest form (Schouten et al. 2009):

$$
R(\rho)=1-2 S\left(\rho_{i}\right) .
$$

where $S\left(\rho_{i}\right)$ is the standard deviation of the response probabilities of responding sample elements under the implemented design. Thus, $\rho_{i}=\rho_{i 5}=\sum_{j=1}^{2} I_{i j 5} p_{i j}$.

As the probabilities used in the R-indicator calculation are those of respondents only, we should simulate the expected responding sample by weighting each element in the gross sample by their predicted probability of response, $\rho_{i}$. Thus, all statistics that would be based on the responding sample are estimated based on the gross sample weighted by $\rho_{i}$.

To optimize the R-indicator we need to minimize $S\left(\rho_{i}\right)$. However, $\rho_{i}$ depends on the allocation $I_{i j 5}$, so to obtain the optimal allocation that will maximize the R-indicator we use an iterative process. We first calculate a starting value for the mean response propensity under Scenario 5 as the mean of the probabilities in each protocol:

$$
\bar{\rho}_{5}^{(1)}=\sum_{i=1}^{n} \sum_{j=1}^{2} p_{i j} / 2 n
$$

Then, for each sample element we compare the distance between $\bar{\rho}_{5}^{(1)}$ and each of the protocol-specific response propensities, $p_{i 1}$ and $p_{i 2}$. The panel member is assigned to the protocol with the smallest distance and this way we obtain allocation for the first iteration:

$$
\begin{gathered}
I_{i 15}^{(1)}=1 \quad \text { and } I_{i 25}^{(1)}=0 \text { if }\left|p_{i 1}-\bar{p}_{5}^{(1)}\right|<\left|p_{i 2}-\bar{p}_{5}^{(1)}\right| ; \\
I_{i 15}^{(1)}=0 \text { and } I_{i 25}^{(1)}=1 \text { otherwise }
\end{gathered}
$$

Each subsequent iteration uses the average probability of participation according to the current allocation as a starting point. Again, distances are compared between this average and the element-specific probabilities to participate in each protocol to see whether some reallocation may improve the R-indicator. In other words, for iteration $(h)$ :

$$
\begin{gathered}
\bar{\rho}_{5}^{(h)}=\sum_{i=1}^{n} \sum_{j=1}^{2} I_{i j 5}^{(h-1)} p_{i j} / n ; \\
I_{i 15}^{(h)}=1 \quad \text { and } \quad I_{i 25}^{(h)}=0 \quad \text { if } \quad\left|p_{i 1}-\bar{p}_{5}^{(h)}\right|<\left|p_{i 2}-\bar{p}_{5}^{(h)}\right| ; \\
I_{i 15}^{(h)}=0 \quad \text { and } \quad I_{i 25}^{(h)}=1 \quad \text { otherwise }
\end{gathered}
$$

Iterations cease when $R(\rho)$ changes very little and very few sample elements are reallocated to a different protocol. Specifically, the Stopping rule is: $0.999<R_{h} / R_{h-1}<$ 1.001 and fewer than one percent of elements switch allocation. 


\subsection{Coefficient of Variation (CV) Optimization}

Variance functions of the nonresponse weights - and therefore of the response propensities - have been proposed as useful measures of the representativity of responding survey samples (Groves et al. 2008; Bethlehem et al. 2011). Schouten et al. (2013) have suggested that the most useful amongst these may be the coefficient of variation of the response propensities. This has the following general form:

$$
C V=\frac{S\left(\rho_{i}\right)}{\bar{\rho}_{i}}
$$

where again $S\left(\rho_{i}\right)$ is the standard deviation of response probabilities amongst the responding sample, and $\bar{\rho}_{i}$ is the mean of these probabilities. A smaller value of CV indicates a more representative sample. To minimize $\mathrm{CV}(k=6)$ we implement the following iterative procedure. For each sample element we first calculate the average of their probabilities to respond in each protocol: $\rho_{i 6}^{(1)}=\left(p_{i 1}+p_{i 2}\right) / 2$. This unrealistic probability serves as a starting value. Based on these average probabilities we simulate the $\mathrm{CV}$ of the responding sample by weighting each element $i$ in the gross sample by $\rho_{i 6}^{(1)}$. Now for each element we estimate two new weighted CVs: keeping the average probabilities for all other sample elements, for element $i$ we substitute $\rho_{i 6}^{(1)}$ with $p_{i 1}$ (CAPI) to estimate $C V_{i 1}^{(1)}$ and then separately we substitute $\rho_{i 6}^{(1)}$ with $p_{i 2}$ (mixed mode) to estimate $C V_{i 2}^{(1)}$. Thus for each element we have two estimates of $\mathrm{CV}$ - one corresponding to allocation of this element to the CAPI protocol and the other to the mixed mode protocol while keeping average probabilities for all other elements. We aim to lower the $\mathrm{CV}$ and therefore at the first iteration we allocate element $i$ to the CAPI single-mode protocol if $C V_{i 1}^{(1)}<C V_{i 2}^{(1)}$ and vice versa.

As a starting point for the second iteration we use the response probabilities based on the protocol allocation after the first iteration. Keeping these probabilities for all other elements, for each element we again estimate the $\mathrm{CV}$ under each of the allocation options, $C V_{i 1}^{(2)}$ and $C V_{i 2}^{(2)}$. Element $i$ will again be allocated to the protocol corresponding to the smaller CV. More iterations can be implemented until there is little or no change in allocation between iterations. Our Stopping rule is that $0.999<C V_{h} / C V_{h-1}<1.001$ and fewer than $1 \%$ of elements switch allocation.

\subsection{ESS (Effective Sample Size) Optimization}

To the best of the authors' knowledge, ESS has not been used as an allocation criterion in the context of adaptive design until now. Yet it has the advantage of reflecting both variance in inclusion probabilities (encompassing both selection probabilities and response probabilities) and the final sample size, both of which influence the precision of survey estimates. Theoretically ESS varies across different estimates, but an approximation proposed by Kish (1965) takes one value for the whole survey, with the general form:

$$
E S S=\frac{\left(\sum_{i=1}^{m} w_{i}\right)^{2}}{\sum_{i=1}^{m} w_{i}^{2}},
$$

where $w_{i}$ is the weight of the respondent $i$ (reciprocal of inclusion probability) and both of the sums are over the $m$ elements in the responding sample. The ESS value indicates the size 
of a fully-responding simple random sample which would provide the same precision as the design in question. Our Scenario 7 therefore involves finding a design which gives the highest value of ESS and therefore the best precision for estimation.

To maximize ESS we again use an iterative procedure. The first step is to use the 'average' response probability for each sample element, $\rho_{i 7}^{(1)}=\left(p_{i 1}+p_{i 2}\right) / 2$ as a starting value to derive 'average' weights, $w_{i 7}^{(1)}=1 / \rho_{i 7}^{(1)}$. For each sample element $i$ we then calculate two weighted ESS values: for the CAPI single-mode protocol and for the mixedmode protocol: $E S S_{i 1}^{(1)}$ uses $1 / p_{i 1}$ for element $i$ and $w_{i 7}^{(1)}$ for each other sample element; and $\operatorname{ESS}_{i 2}^{(1)}$ uses $1 / p_{i 2}$ for element $i$ and $w_{i 7}^{(1)}$ for each other sample element. As the ESS depends on the inclusion probabilities of the responding sample we simulate it by weighting each of the $n$ elements in the gross sample by their probability to respond ( $p_{i 1}$ or $p_{i 2}$ for element $i$ and $\rho_{i 7}^{(1)}$ for each other element). Element $i$ is allocated to the CAPI protocol if $E S_{i 1}>E S S_{i 2}$, and vice versa.

Each subsequent iteration, $(h)$, uses the weights from the previous iteration as starting values and analogously calculate $E S S_{i 1}^{(h)}$ and $E S S_{i 2}^{(h)}$ for each sample element $i$, reallocating the element if their current assignment achieves lower ESS than assignment to the other protocol. Similarly to the previously-described procedures, our Stopping rule is that $0.999<E S S_{h} / E S S_{h-1}<1.001$ and fewer than one percent of elements switch allocation.

All optimization and analysis is implemented in Stata 14.1.

\section{Data Collection Costs}

When the alternative data collection protocols differ in terms of costs, it is important to take this into account when selecting an allocation method. Broadly speaking, there are two ways to do this. The first is to explicitly incorporate data collection costs into the allocation criteria, perhaps either by applying a cost constraint or by extending the criteria themselves to include a cost element. The second way is to include a prediction of data collection costs in the evaluation of alternative allocation scenarios. Here, we do the latter.

Data collection costs for any survey can be expressed in terms of the fixed and variables costs associated with various aspects of the design. The traditional model (Groves 1989, p.51) can be extended to allow any of the fixed or variable cost components to vary between design options and hence to facilitate the comparison of designs. Costs may vary between the scenarios we consider here due to differences in the number of sample elements attempted, and achieved, in each mode, and (large) differences between modes in the unit costs of data collection. We can express the data collection cost as follows:

$$
C_{k}=C_{0}+\sum_{l=1}^{2}\left[C_{0 l}+C_{1 l} n_{k l}+C_{2 l} m_{k l}\right],
$$

where

$C_{0}$ represents fixed costs that are incurred regardless of which data collection protocols are implemented and regardless of sample sizes;

$C_{0 l}$ are fixed costs associated with including mode $l$ in the survey protocol $(l=1$ for CAPI, 2 for web); 
$C_{1 l}$ is the unit cost per sample element attempted in mode $l$;

$C_{2 l}$ is the additional unit cost per sample element that responds in mode $l$;

$n_{k l}$ is the number of sample elements attempted in mode $l$ under scenario $k$;

$m_{k l}$ is the number of sample elements that respond in mode $l$ under scenario $k$.

Note that in our set-up $n_{k 1}=\sum_{i=1}^{n}\left[I_{i 1 k}+I_{2 k}\left(1-q_{i 2}\right)\right]$, as elements allocated to the mixed mode protocol will be attempted by CAPI if they do not respond by web in the first fieldwork phase, while $n_{k 2}=\sum_{i=1}^{n} I_{2 k}$. The mode-specific responding numbers are as follows: $m_{k 1}=\sum_{i=1}^{n}\left[I_{i 1 k} p_{i 1}+I_{2 k}\left(p_{i 2}-q_{i 2}\right)\right] ; \quad m_{k 2}=\sum_{i=1}^{n} I_{i 2 k} q_{i 2}$. The unit cost per sample element attempted by CAPI $\left(C_{11}\right)$ includes interviewer time and travel expenses to contact and liaise with the sample element, plus associated tasks and materials such as the mailing of advance letters. The unit cost per sample element attempted by web $\left(C_{12}\right)$ includes the costs of invitation and reminder mailings and emailings. The additional unit cost per sample element responding by CAPI $\left(C_{21}\right)$ includes interviewer time for carrying out the interview, plus costs of data editing and data management, while the additional unit cost per sample element responding by web $\left(C_{22}\right)$ consists solely of the costs of data editing and data management. The fixed costs associated with a CAPI fieldwork operation apply to all seven of our scenarios, so we can subsume these within $C_{0}$ and set $C_{01}=0$, leaving $C_{02}$ to represent the margin additional cost of setting up and running a web survey option. For any given values of the cost parameters $\left(C_{0}, C_{02}, C_{11}, C_{12}, C_{21}, C_{22}\right)$ and gross sample size $n, C_{k}$ therefore depends on the allocation to protocols, the protocol-specific response rates and the proportion of mixed-mode respondents who respond by web.

\section{Prediction of Outcomes}

To assess the relative performance of each of the allocation scenarios, we predict several quality measures for each scenario as well as estimating the data collection cost. The quality measures correspond to the four measures that were optimized under the adaptive scenarios - overall response rate, R-indicator, CV, and ESS - plus the proportion of the total sample responding by web, as this indicates the relative cost of data collection.

For each of the six scenarios, the total response rate for scenario $k$ is predicted as the mean of the predicted element response propensities:

$$
\bar{p}_{k}=\frac{1}{n} \sum_{i=1}^{n} \sum_{j=1}^{2} I_{i j k} p_{i j}=\frac{1}{n} \sum_{i=1}^{n} \rho_{i k}
$$

For example, the predicted response rate for the CAPI-for-all scenario is:

$$
\bar{p}_{1}=\frac{1}{n} \sum_{i=1}^{n} \sum_{j=1}^{2} I_{i j 1} p_{i j}=\frac{1}{n} \sum_{i=1}^{n} I_{i 11} p_{i 1}
$$

The web response rate for the total sample is predicted for scenario $k$ thus:

$$
\bar{q}_{k}=\frac{1}{n} \sum_{i=1}^{n} I_{i 2 k} q_{i 2}
$$


The web response rate within the sample allocated to the mixed-mode protocol is predicted as follows:

$$
\overline{q c}_{k}=\frac{\sum_{i=1}^{n} I_{i 2 k} q_{i 2}}{\sum_{i=1}^{n} I_{i 2 k}}
$$

The other three outcome measures that we consider - R-indicator, CV, and ESS - are all characteristics of the responding sample and are predicted by weighting each member of the gross sample by the model-predicted response probability for the mode to which they are allocated under that scenario, $\rho_{i k}$. The method of calculation for each of the predicted quantities is presented in the Appendix.

Data collection costs are estimated by assuming the following relative values for the cost components. We believe these to be broadly realistic, at least for the UK context, assuming that fieldwork is carried out by an established survey agency: $C_{0}=20,000, C_{02}=8,000, C_{11}=20, C_{12}=1, C_{21}=20, C_{22}=0.5$. In practice, the relative size of these cost components will depend on features such as the complexity of the survey instruments and the software and methods used for script writing and testing. We present the relative costs of each allocation scenario for three alternative gross sample sizes of $n=2,000,10,000$, and 40,000.

\section{Results}

Table 2 presents predicted outcomes for each of the four adaptive design scenarios and for each of the two non-adaptive scenarios in which all sample elements are allocated to a single protocol. The final column presents the observed outcomes for the experimental allocation to protocols that was implemented at IP Wave 5.

For each scenario, the first two rows of Table 2 present the percentages of sample elements allocated to each of the two data collection protocols, $\frac{100}{n} \sum_{i=1}^{n} I_{i 1 k}$ and $\frac{100}{n} \sum_{i=1}^{n} I_{i 2 k}$ respectively. The third and fourth row present the response rate among those allocated to the CAPI protocol (i.e., their average CAPI probabilities to participate) and the response rate among those allocated to the mixed-mode protocol, respectively. The fifth row presents the web response rate amongst people allocated to the mixed-mode protocol, $\overline{q c}_{k}$, while the sixth row presents the proportion of the whole sample who respond by web, $\bar{q}_{k}$ (which is the product of the proportion allocated to the mixed-mode protocol and the web response rate among them). Row 7 presents the total expected response rate (average of response probabilities for the specified allocation), $\bar{p}_{k}$, while the following three rows present the R-indicator $\left(R_{k}\right)$; CV $\left(C V_{k}\right)$; and ESS $\left(E S S_{k}\right)$. Additionally we present the responding sample size (resulting $n$ ), which is a product of the 2,109 gross sample size and $\bar{p}_{k}$, and the estimated design effect, calculated as the responding sample size divided by ESS. Note that except for the last column ('experimental allocation') all these outcome values are predicted based on the model-predicted values of participation propensity, as explained in Section 5 above. The final three rows of the table present estimated data collection costs relative to the benchmark CAPI-only scenario (Scenario 1) for three alternative sample sizes. 


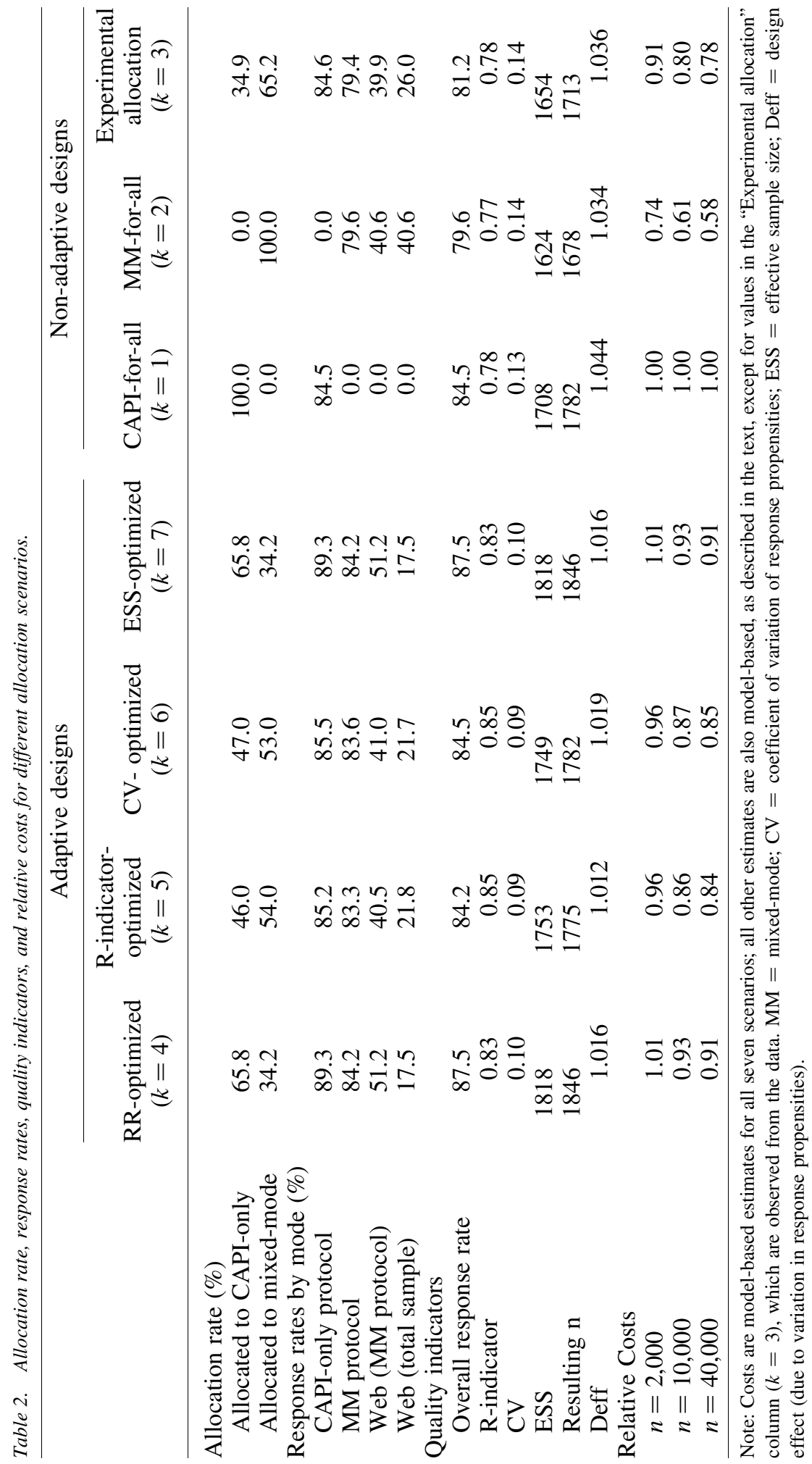


Our interest is primarily in comparing the four adaptive designs and in comparing each of them with the two single-protocol designs. The two single-protocol designs can in that sense be seen as benchmarks. We therefore describe the benchmark designs first. If all sample elements were allocated to the CAPI protocol ('CAPI-for-all' scenario, Table 2) the predicted response rate would be $84.5 \%$ with an R-indicator of 0.78 and an effective sample size of 1,708 . If, on the other hand, all sample elements were allocated to the mixed-mode protocol ('MM-for-all' scenario) the predicted response rate would be lower (79.6\%), as would the effective sample size $(1,624)$ but the R-indicator would be similar $(\mathrm{R}=0.77)$. If only single-protocol designs were to be considered, it would most likely be concluded that the CAPI protocol was slightly superior to the mixed-mode protocol.

Comparing first the proportions allocated to each protocol, it can be seen that two scenarios - the scenario in which allocation is based on maximizing response rate ('RRoptimized' scenario in Table 2) and the scenario in which allocation aims to maximize the effective sample size ('ESS-optimized') - result in an identical allocation, in which $65.8 \%$ of sample elements are allocated to the CAPI single-mode protocol and $34.2 \%$ to the mixed-mode protocol. This may not be true in general but in our situation selecting respondents with higher probabilities was more important to ESS optimization. The proportion allocated to the mixed-mode protocol is substantially higher in the other two scenarios, where the aim is to maximize representativity: $53.0 \%$ when the aim is to minimize the coefficient of variation of the response propensities ('CV-optimized') and $54.0 \%$ when the aim is to maximize the $\mathrm{R}$-indicator (' $\mathrm{R}$-indicator-optimized').

In terms of the four quality measures of interest, it is striking that performance varies only slightly between the four adaptive designs. While the two scenarios that aim to maximize representativity do indeed perform better on both representativity measures than the RR/ESS scenario, differences appear to be small: the R-indicator is predicted to be 0.85 in both the CV-optimized and R-indicator scenarios, but 0.83 in the RR/ESS scenario, while the coefficient of variation of the response probabilities is 0.09 in both the CVoptimized scenario and the R-indicator scenario, compared to 0.10 in the RR/ESS scenario. The RR/ESS scenario, on the other hand, appears to perform slightly better in terms of both response rate and effective sample size. The effective sample size is 1,818 under the RR/ESS scenario, compared to 1,753 and 1,749 under the R-indicator and CVoptimized scenarios respectively. Predicted response rate is $87.5 \%$ under the RR/ESS scenario, compared to response rates of $84.2 \%$ and $84.5 \%$ respectively for the R-indicator and CV-optimized scenarios (and $84.5 \%$ for the CAPI-for-all scenario).

Comparing the adaptive designs with the single-protocol designs, we see that for each of the four outcome indicators, the design that performs best is one of the adaptive designs. Indeed, for three of the four indicators all four of the adaptive designs outperform both of the single- protocol designs. The sole exception is response rate, where the $\mathrm{CV}$-optimized and R-indicator scenarios perform similarly to the CAPI single-mode protocol. It is therefore clear that adaptive designs can out-perform single-protocol designs and that in this study maximized performance in terms of one measure tends to coincide with improved performance on other measures too.

One other important aspect is the cost of data collection. Compared to the CAPI-only design, we see that cost savings with the adaptive designs are minimal or non-existent when the gross sample sample is 2,000, but larger, though still modest, with larger sample 
sizes. With the larger sample sizes, the relative costs are largely driven by the proportion of the total sample who respond by web (row 6 of Table 2) as in a sequential mixed-mode design such people will not need a visit by an interviewer (reflected in the size of $C_{12}$ relative to $C_{11}$ and $C_{22}$ relative to $C_{21}$ ). But with the smaller sample size, much of this saving is eroded by the additional fixed cost associated with offering two modes $\left(C_{02}\right)$. Naturally, the MM-for-all scenario results in the highest overall web response rate $(40.6 \%)$ and hence the lowest data collection costs. Yet this is the allocation scenario that performs worst on all four of the outcome indicators of interest (lowest total response rate, lowest R-indicator, highest CV, and lowest ESS). The overall web response rate is lower in the adaptive designs and hence data collection costs would be higher. Costs would be slightly lower with the R-indicator and CV-optimized scenarios (web response rate $21.7 \%$ $21.9 \%$ ) than with the RR/ESS-optimized scenario (17.5\%).

\section{Conclusions}

A few important conclusions emerge from our findings. First and foremost we find that through adaptive design allocation, response rate, representativity (R-indicator and CV), and efficiency (ESS and deff) can all be improved. All adaptive designs resulted in almost the same or better overall response rate, and consistently better R-indicator, CV, ESS and deff than either of the one-protocol-for-all designs or the random allocation observed in the experiment. This suggests a promising future for adaptive design within survey research: even the best non-adaptive designs might benefit from a design review.

For example, in many contexts for a long time researchers have believed that the CAPIfor-all design achieves the highest response rate. Yet our study shows that it is possible to improve response rate from $84.5 \%$ for the CAPI-for-all design to $87.5 \%$ for the RR/ESSoptimized adaptive design. Current best practice in survey design often points to use of the best one-protocol-for-all design without considering whether such a design may be outperformed by an adaptive design.

Second, we did not find a single allocation scenario that was optimal for all outcome criteria. Some designs are predicted to achieve a higher response rate (RR-optimized) while others should achieve higher representativity (R-indicator and CV optimized). But variation in response rates, representativity measures (R-indicator and $\mathrm{CV}$ ) and precision of estimation (ESS) is small between the adaptive designs considered in this study. While it is clear that adaptive designs achieve better quality outcomes than non-adaptive designs, it is harder to select the best scenario among them. Our original intuition was that ESSoptimized design should be preferable as it should come closest to the most efficient design. It is interesting that in our study the ESS-optimized design achieved the same allocation as RR-optimized design. This may not necessarily be the case in general, but it could be the case that if the variation in response propensities does not differ greatly between allocation scenarios the responding sample size - which is determined by response rate - becomes the dominant influence on the effective sample size. We feel this should be explored further, especially given the relative ease of implementing RR-optimized design.

We must also note a limitation of our empirical results, which is that the allocation models and the outcomes are not based on independent samples. We only had one sample available, and to have randomly split it into test and control samples would have done severe damage 
to statistical power and precision. In consequence, there is potential for bias in our findings. In due course, as researchers begin to allocate survey samples based on models from other surveys, it should be possible for future research to overcome this limitation.

We have demonstrated how alternative allocation criteria can be applied, but to do this we have benefited from the rich context provided by the Understanding Society Innovation Panel. Specifically, to implement our methods it is necessary, a) to have informative auxiliary data available for all elements in the gross sample and, b) to have an applicable model of predicted response propensity in terms of these auxiliary data. Most types of panel surveys, from Wave 2 onwards, typically meet the requirement of rich auxiliary data. Cross-sectional studies may be able to draw upon sampling frame data, linked data, or interviewer observations. Requirement b) implies that the researcher must have confidence that models which, by necessity, must be based on prior studies, are likely to be applicable to the current survey. Such models need not necessarily be based on randomised methodological experiments such as the one we have presented here. They could instead be based on similar surveys each using a different protocol of interest. However, in either case the surveys must have broad external validity.

An extension of our allocation criteria, for example to include cost constraints, would be possible. For example, a researcher may be interested in optimizing one of the criteria that we have presented, but with a constraint that the data collection cost should not exceed a certain proportion of the costs of a CAPI-for-all design. The cost model that we have introduced in Section 4, or a variant of it, could be used for this purpose. With the data collection protocols that we have considered in this article, such a constraint would have the effect of ensuring that a certain minimum proportion of respondents participate online (and this proportion would depend on the overall sample size). Extensions of this kind to the allocation criteria, effectively involving simultaneously meeting multiple criteria, would seem a natural next step.

\section{Appendix}

\section{Prediction of Quality Measures}

For each scenario, the R-indicator, CV, and ESS are predicted by adapting the standard formula for the respective quantities to reflect the fact that we are using information from the gross sample rather than the net (responding) sample. In each case, wherever a summation is made over sample elements, instead of an unweighted sum of responding sample elements we substitute a weighted sum of gross sample elements, where the weight represents the probability of the element appearing in the responding sample, in other words $\rho_{i k}$. The predicted outcomes for scenario $k$ are therefore as follows.

R-indicator:

$$
\hat{R}_{k}=1-2 \hat{s}_{k}
$$

where

$$
\hat{s}_{k}=\sqrt{\frac{1}{n-1} \sum_{i=1}^{n} \rho_{i k}\left(\rho_{i k}-\bar{p}_{k}\right)^{2}}
$$




$$
=\sqrt{\frac{1}{n-1} \sum_{i=1}^{n} \sum_{j=1}^{2} I_{i j k} p_{i k}\left(p_{i k}-\bar{p}_{k}\right)^{2}}
$$

Coefficient of variation of the response propensities (' $\mathrm{CV}$ '):

$$
\widehat{C V}_{k}=\frac{\hat{s}_{k}}{\bar{p}_{k}}
$$

Effective sample size ('ESS'):

$$
\begin{aligned}
\widehat{E S S}_{k} & =\frac{\left(\sum_{i=1}^{n} \rho_{i k}\left(1 / \rho_{i k}\right)\right)^{2}}{\sum_{i=1}^{n} \rho_{i k}\left(1 / \rho_{i k}\right)^{2}} \\
& =\frac{n^{2}}{\sum_{i=1}^{n}\left(1 / \rho_{i k}\right)}
\end{aligned}
$$

Data collection costs:

$$
\begin{aligned}
\hat{C}_{k}= & C_{0}+J_{k} C_{02}+C_{11} \sum_{i=1}^{n}\left[I_{i 1 k}+I_{2 k}\left(1-q_{i 2}\right)\right]+C_{21} \sum_{i=1}^{n}\left[I_{i 1 k} p_{i 1}+I_{2 k}\left(p_{i 2}-q_{i 2}\right)\right] \\
& +C_{12} \sum_{i=1}^{n} I_{2 k}+C_{22} \sum_{i=1}^{n} I_{i 2 k} q_{i 2},
\end{aligned}
$$

where

$$
J_{k}=0 \quad \text { if } \quad k=1 ; \quad J_{k}=1 \quad \text { if } \quad 2 \leq k \leq 7
$$

\section{References}

Al Baghal, T. (ed.). 2014. "Understanding Society Innovation Panel Wave 6: Results from Methodological Experiments.” Understanding Society Working Paper Series 2014-04. Available at: https://www.understandingsociety.ac.uk/research/publications/workingpaper/understanding-society/2014-04.pdf (accessed 24 February 2016).

Al Baghal, T. (ed.). 2015. "Wave 7: Results from Methodological Experiments." Understanding Society Working Paper Series 2015-03. Available at: https://www. understandingsociety.ac.uk/research/publications/working-paper/understandingsociety/2015-03.pdf (accessed 24 February 2016).

Bethlehem, J., F. Cobben, and B. Schouten. 2011. Handbook of Nonresponse in Household Surveys. New Jersey: Wiley.

Burton, J. (ed.). 2013. "Understanding Society Innovation Panel Wave 5: Results from Methodological Experiments." Understanding Society Working Paper Series 2013-06. Available at: https://www.understandingsociety.ac.uk/research/publications/workingpaper/understanding-society/2013-06.pdf (accessed 24 February 2016).

Calinescu, M. and B. Schouten. 2015. "Adaptive Survey Designs to Minimize Survey Mode Effects - a Case Study on the Dutch Labor Force Survey." Survey Methodology 41(2): 403-425. 
Durrant, G., R.M. Groves, L. Staetsky, and F. Steele. 2010. "Effects of Interviewer Attitudes and Behaviors on Refusal in Household Surveys." Public Opinion Quarterly 74(1) : 1-36. Doi: http://dx.doi.org/10.1093/poq/nfp098.

Durrant, G.B., O. Maslovskaya, and P. Smith. 2015. "Modelling Final Outcome and Length of Call Sequence to Improve Efficiency in Interviewer Call Scheduling." Journal of Survey Statistics and Methodology 3: 397-424. Doi: http://dx.doi.org/10. 1093/jssam/smv008.

Groves, R.M. 1989. Survey Errors and Survey Costs. New York: Wiley.

Groves, R.M., J.M. Brick, W. Kalsbeek, B. Harris-Kojetin, F. Kreuter, B.-E. Pennell, T. Raghunathan, B. Schouten, T. Smith, R. Tourangeau, A. Bowers, M. Jans, C. Kennedy, R. Levenstein, K. Olson, E. Peytcheva, S. Ziniel, and J. Wagner. 2008. "Issues Facing the Field: Alternative Practical Measures of Representativeness of Survey Respondent Pools." Survey Practice 1(3): 1-6.

Groves, R.M. and M.P. Couper. 1998. Nonresponse in Household Interview Surveys. New York: Wiley.

Groves, R.M. and S.G. Heeringa. 2006. "Responsive Design for Household Surveys: Tools for Actively Controlling Survey Errors and Costs." Journal of the Royal Statistical Society Series A 169: 439-457.

Groves, R.M. and E. Peytcheva. 2008. "The Impact of Nonresponse Rates on Nonresponse Bias: A Meta-Analysis.” Public Opinion Quarterly 72(2): 167-189. Doi: http://dx.doi. org/10.1093/poq/nfn011.

Jäckle, A., P. Lynn, and J. Burton. 2015. "Going Online with a Face-to-Face Household Panel: Effects of a Mixed Mode Design on Item and Unit Nonresponse." Survey Research Methods 9(1): 57-70. Doi: http://dx.doi.org/10.18148/srm/2015.v9i1.5475.

Kanuk, L. and C. Berenson. 1975. "Mail Surveys and Response Rates: A Literature Review." Journal of Marketing Research 12: 440-453.

Kaplowitz, M.D., T.D. Hadlock, and R. Levine. 2004. "A Comparison of Web and Mail Survey Response Rates.” Public Opinion Quarterly 68: 94-101.

Kaplowitz, M.D., F. Lupi, M.P. Couper, and L. Thorp. 2012. "The Effect of Invitation Design on Web Survey Response Rates." Social Science Computer Review 30(3): 339-349. Doi: http://dx.doi.org/10.1177/0894439311419084.

Kish, L. 1965. Survey Sampling. New York: Wiley.

Lundquist, P. and C.-E. Särndal. 2013. "Aspects of Responsive Design with Applications to the Swedish Living Conditions Survey." Journal of Official Statistics 29(4): 557-582. Doi: https://doi.org/10.2478/jos-2013-0040.

Lynn, P. 2014. "Targeted Response Inducement Strategies on Longitudinal Surveys.” In Improving Survey Methods: Lessons from Recent Research, edited by U. Engel, B. Jann, P. Lynn, A. Scherpenzeel, and P. Sturgis, pp. 322-338. Abingdon UK: Psychology Press. Lynn, P. 2017. "From Standardised to Targeted Survey Procedures for Tackling NonResponse and Attrition." Survey Research Methods 11(1): 93-103. Doi: http://dx.doi. org/10.18148/srm/2017.v11i1.6734.

Lynn, P. and A. Jäckle. in press. "Mounting Multiple Experiments on Longitudinal Social Surveys: Design and Implementation Considerations." In Experimental Methods in Survey Research: Techniques that Combine Random Sampling with Random 
Assignment, edited by P.J. Lavrakas, M.W. Traugott, C. Kennedy, A.L. Holbrook, E.D. De Leeuw, and B.T. West. Hoboken NJ: Wiley.

Särndal, C.-E. 2011. "The 2010 Morris Hansen Lecture: Dealing with Survey Nonresponse in Data Collection, in Estimation.” Journal of Official Statistics 27(1): 1-21.

Schouten, B., J. Bethlehem, K. Beullens, O. Kleven, G. Loosveldt, A. Luiten, K. Rutar, N. Shlomo, and C. Skinner. 2012. "Evaluating, Comparing, Monitoring, and Improving Representativeness of Survey Response Through R-Indicators and Partial RIndicators." International Statistical Review 80(3): 382-399.

Schouten, B., M. Calinescu, and A. Luiten. 2013. "Optimizing Quality of Response Through Adaptive Survey Designs.” Survey Methodology 39(1): 29-58.

Schouten, B., F. Cobben, and J. Bethlehem. 2009. "Indicators for the Representativeness of Survey Response.” Survey Methodology 35(1): 101-113.

Schouten, B. and N. Shlomo. 2015. "Selecting Adaptive Survey Design Strata with Partial R-indicators.” International Statistical Review 85(1): 143-163. Doi: http://dx.doi.org/ 10.1111/insr.12159.

Schouten, B., N. Shlomo, and C. Skinner. 2011. "Indicators for Monitoring and Improving Representativeness of Response.” Journal of Official Statistics 27(2): 231-253.

Sheehan, K.B. 2001. "Email Survey Response Rates: A Review." Journal of ComputerMediated Communication 6(2): 1-16.

Shlomo, N., B. Schouten, and V. de Heij. 2013. Designing Adaptive Survey Designs with $R$-Indicators. Available at: http://www.risq-project.eu/papers/shlomo-schouten-heij2013.pdf (accessed February 24 2016).

Singer, E. and C. Ye. 2013. "The Use and Effects of Incentives in Surveys." Annals of the American Academy of Political and Social Science 645: 112.

Uhrig, S.C. 2008. The Nature and Causes of Attrition in the British Household Panel Study. ISER Working Paper Series, 2008(05). Colchester: Institute for Social and Economic Research, University of Essex.

Wagner, J.R. 2008. Adaptive Survey Design to Reduce Nonresponse Bias, PhD Dissertation. University of Michigan.

Wagner, J.R. 2012. "Research Synthesis: a Comparison of Alternative Indicators for the Risk of Nonresponse Bias." Public Opinion Quarterly 76(3): 555-575. Doi: http://dx. doi.org/10.1093/poq/nfs032.

Watson, N. and M. Wooden. 2009. "Identifying Factors Affecting Longitudinal Survey Response.” In Methodology of Longitudinal Surveys, edited by P. Lynn, pp. 157-181. Chichester: Wiley. Doi: http://dx.doi.org/10.1002/9780470743874.

White, N., T. Bourne, and A. Patterson. 2011. Understanding Society Innovation Panel 2. Technical Report. Available at: https://www.understandingsociety.ac.uk/documentation/ innovation-panel/technical-reports (accessed January 24 2017).

$\mathrm{Yu}$, J. and H. Cooper. 1983. "A Quantitative Review of Research Design Effects on Response Rates to Questionnaires.” Journal of Marketing Research 20(1): 36-44.

Received March 2016

Revised June 2017

Accepted June 2017 